\title{
CARGAS ELEVADAS DE TREINAMENTO ALTERAM FUNÇÕES COGNITIVAS EM JOGADORES DE FUTEBOL
}

\author{
HIGH LOADS OF TRAINING AFFECT COGNITIVE FUNCTIONS IN SOCCER PLAYERS
}

CARGAS ELEVADAS DE ENTRENAMIENTO ALTERAN FUNCIONES COGNITIVAS EN JUGADORES DE FÚTBOL

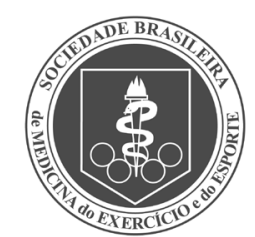

Artigo Original
Felipe de Oliveira Matos ${ }^{1,3}$

(Educador Físico)

Dietmar Martin Samulski ${ }^{1}$ (Educador Físico)

Jorge Roberto Perrout de Lima² (Educador Físico)

Luciano Sales Prado ${ }^{1}$

(Educador Físico)

1. Universidade Federal de Minas Gerais, Belo Horizonte, MG, Brasil. 2. Universidade Federal de Juiz de Fora, Juiz de Fora, MG, Brasil. 3. Universidade Estadual de Maringá, Maringá, PR, Brasil.

\section{Correspondência:}

Praça independência, 385, Centro. Ivaiporã, PR, Brasil. 86870-970. felipeomatos@yahoo.com.br

\section{RESUMO}

Introdução: No esporte de rendimento, altas cargas de treinamento são necessárias para que ocorram adaptações biológicas que levem ao aprimoramento da forma física e do desempenho esportivo. Objetivo: Investigar o comportamento de variáveis psicológicas e fisiológicas, possíveis indicadores dos estados de estresse e recuperação, em jogadores de futebol ao longo da temporada esportiva, e a possibilidade da utilização desses como marcadores dos estados de treinabilidade dos atletas para prevenir quedas do desempenho e o overtraining. Métodos: Participaram deste estudo 14 jogadores de futebol (18,4 \pm 0,6 anos, 9,64 $\pm 1,61 \%$ com gordura e $\left.V_{2 \text { máx }} 49,46 \pm 2,88 \mathrm{ml} / \mathrm{kg} / \mathrm{min}\right)$ da categoria júnior de uma equipe de primeira divisão do Brasil. Foram monitorados a percepção de estresse e a recuperação por meio do questionário RESTQ-Sport, a variabilidade de frequência cardíaca (VFC) de repouso, analisada no domínio do tempo e da frequência, e o tempo de reação simples. Efetuaram-se três coletas de dados, um controle (C), outra após quatro semanas de treinamentos intensos (T1), e finalmente depois de quatro semanas de treinamentos leves (T2) realizados após T1. Resultados: Não foi encontrada diferença significativa em nenhuma das escalas do RESTQ-Sport e na VFC nos três momentos de coletas. O tempo de reação simples apresentou um aumento significativo $(p=0,047)$ em T1 em relação a C. Conclusão: Os resultados indicam que o tempo de reação foi a única variável sensível às alterações do treinamento estudado, podendo ser considerado um bom indicador da treinabilidade dos atletas.

Palavras-chave: estresse fisiológico, frequência cardíaca, recuperação de função fisiológica, tempo de reação.

\section{ABSTRACT}

Introduction: In the performance sports, high training loads are necessary for biological adaptations to occur leading to the improvement of fitness and sports performance. Objective: To investigate the behavior of the psychological and physiological variables, indicators of possible states of stress and recovery in soccer players throughout the sports season, and the possibility of using these as markers of the conditions of athletes trainability to prevent decreased performance and overtraining. Methods: The study included 14 soccer players $\left(18.4 \pm 0.6\right.$ years, $9.64 \pm 1.61 \%$ with fat and $\mathrm{VO}_{2 \text { máx }} 49.46 \pm$ $2.88 \mathrm{~m} / \mathrm{kg} / \mathrm{min}$ ) of a junior class of a team of the first division of Brazil. The perception of stress and recovery, the heart rate variability (HRV) at rest, analyzed in the time and frequency domain, and simple reaction time were monitored through the questionnaire RESTQ-Sport. We carried out three collections of data, a control (C), another after four weeks of intense training (T1), and finally after four weeks of light training (T2) performed after T1. Results: No significant difference was found in any of the scales of RESTQ-Sport and HRV at the three collections. The simple reaction time showed a significant increase ( $p=0.047$ ) in $T 1$ compared to $C$. Conclusion: The results indicate that the reaction time was the only variable sensitive to changes in the training study and could be considered a good indicator of trainability of athletes.

Keywords: stress, physiological, heart rate, recovery of function, reaction time.

\section{RESUMEN}

Introducción: En el deporte de rendimiento, las altas cargas de entrenamiento son necesarias para que ocurran adaptaciones biológicas que lleven a la mejora de la forma física y del desempeño deportivo. Objetivo: Investigar el comportamiento de variables psicológicas y fisiológicas, posibles indicadores de los estados de estrés y recuperación, en jugadores de fútbol a lo largo de la temporada deportiva, y la posibilidad del uso de los mismos como marcadores de los estados de entrenabilidad de los atletas para prevenir caídas del desempeño y overtraining. Métodos: Participaron en este estudio 14 jugadores de fútbol (18,4 $\pm 0,6$ años, 9,64 $\pm 1,61 \%$ con gordura y $\left.V_{2 \text { máx }} 49,46 \pm 2,88 \mathrm{~m} / \mathrm{kg} / \mathrm{min}\right)$ de la categoría junior de un equipo de primera división de Brasil. Fueron monitoreadas la percepción de estrés y la recuperación por medio del cuestionario RESTQ-Sport, la variabilidad de frecuencia cardíaca (VFC) de reposo, analizada en el dominio del tiempo y de la frecuencia, y el tiempo de reacción simple. Se efectuaron tres colectas de datos, un control (C), otra después de cuatro semanas de entrenamientos intensos (T1), y finalmente después de cuatro semanas de entrenamientos leves (T2) realizados después de T1. Resultados: No fue encontrada diferencia significativa en ninguna de las escalas de RESTQ-Sport y en VFC en los tres momentos de colecta. El tiempo de reacción simple presentó un aumento significativo $(p=0,047)$ en T1 en relación a C. Conclusión: Los resultados indican que el tiempo de reacción fue la única variable sensible a las alteraciones del entrenamiento estudiado, pudiendo ser considerado un buen indicador de la entrenabilidad de los atletas.

Palabras clave: estrés fisiológico, frecuencia cardíaca, recuperación de función fisiológica, tiempo de reacción. 


\section{INTRODUÇÃO}

No esporte de rendimento, altas cargas de treinamento se fazem necessárias para que ocorram adaptações biológicas, as quais levem ao o aprimoramento da forma física e do desempenho esportivo ${ }^{1,2}$. Atletas são frequentemente submetidos a altas cargas de treinamento (duração, intensidade e frequência dos estímulos) ${ }^{3}$, entendendo-se carga de treinamento como um estímulo funcional, capaz de provocar distúrbios na homeostase (equilíbrio dinâmico) de células, tecidos e órgãos, causando adaptações físicas e/ou psicológicas ${ }^{4}$.

Uma estratégia usual no esporte de alto rendimento é a alternância de períodos de altas e baixas cargas de treinamento na busca do aprimoramento da forma física ${ }^{3}$. Após um período de cargas elevadas de treinamento pode haver um declínio do desempenho ${ }^{5}$ o qual, com a recuperação devida, retorna a níveis similares ou superiores de rendimento ${ }^{1,6}$. Entretanto, essa elevação nas exigências do treinamento pode não somente gerar adaptações positivas nos atletas e em seu desempenho, como também trazer prejuízos ao desportista quando o treinamento exceder a capacidade de regeneração individual, causando estagnação e/ou queda do rendimento e podendo levar a um quadro conhecido como "síndrome do excesso de treinamento" ou overtraining $1,2,4,5,7,8$.

O overtraining é considerado um desequilíbrio entre as cargas de treinamento e a devida recuperação, capaz de regenerar o organismo ${ }^{6}$, assim como é o resultado de um desequilíbrio entre estresse e recuperação, no qual o estresse é resultado de fatores inter e extra-treinamento, como carga de treinamento, número excessivo de competições, frequentes viagens, lesões, dificuldades financeiras, conflitos pessoais e dificuldades de relacionamento (pais, amigos, familiares, técnicos) ${ }^{6,9}$.

Pesquisas tentam identificar marcadores, possíveis indicadores fisiológicos e bioquímicos do estado de treinamento, tais como relação testosterona/cortisol, concentrações máximas de lactato, concentrações plasmáticas de catecolaminas, hemoglobina, creatina quinase $\mathrm{e}^{4,10-12}$ e o balanço simpáto-vagal, o qual tem sido constantemente investigado com a utilização da variabilidade da frequência cardíaca em atletas com overtraining e em treinamento, sob diferentes cargas de treinamento, devido à grande influência do treinamento sobre o controle autonômico cardíaco 9,13-17. No entanto, os resultados encontrados ainda se mostram inconsistentes na literatura, pois alterações nessas variáveis acontecem não apenas quando os indivíduos estão excedendo o treinamento, mas também durante períodos de treinos intensos ${ }^{6,18}$, os quais originam um estado de alarme no organismo caracterizado como "overreaching" ou "overtraining funcional", o qual necessita de até duas semanas de recuperação para o restabelecimento da forma física ${ }^{7}$, sendo necessário para a melhora da capacidade aeróbica, cardiovascular e do desempenho. Aparentemente, variáveis fisiológicas se comportam de maneiras diferentes dependendo do tipo de treinamento; volume, frequência, intensidade, demanda de rendimento e tipo de modalidade praticada, além de mostrarem grandes diferenças inter-indivíduos.

Outro problema observado quanto à utilização de variáveis fisiológicas e bioquímicas são os altos custos e a demora nas análises laboratoriais, o que inviabiliza uma rápida avaliação da situação e busca por soluções, e, consequentemente, a intervenção nos programas de treinamento ${ }^{18}$. Sendo assim, muitos estudos têm buscado avaliar as cargas de treinamento por meio de instrumentos psicométricos, tais como o Perfil dos Estados de Humor - POMS 1,7,19, e o Questionário de Estresse e Recuperação para Atletas - RESTQ-Sport 5,7,8,20,21, que embora se mostrem sensíveis e expressivos, ainda apresentam resultados pouco consistentes, quando analisados de maneira isolada, além de possuírem baixa reprodutibilidade nos treinamentos diários ${ }^{1}$, devido à possibilidade de efeito de aprendizagem e grande número de itens, o que poderia causar desmotivação ao responder o questionário, originando respostas não fidedignas.

Portanto, ainda não se tem conhecimento de um marcador objetivo, preciso e confiável, de aplicação prática e simplificada para o monitoramento do impacto da carga de treinamento sobre aspectos psicofisiológicos de atletas competitivos. Nederhof et al. ${ }^{6}$ levantam a hipótese do tempo de reação ser uma ferramenta útil para a avaliação dos estados de treinamento de atletas baseados no aumento do tempo de resposta de indivíduos com fadiga crônica e depressão e as singularidades dessas patologias com o overtraining. Outros estudos evidenciam essa possibilidade ao se referirem a prejuízos nas funções cognitivas em indivíduos com overtraining ou submetidos a elevadas cargas de treinamento $0^{1,4,6,7,15,19}$.

No entanto, poucos são os estudos que relacionaram o desempenho cognitivo com as cargas de treinamento 1,15. Assim, o presente estudo busca avaliar o tempo de reação em conjunto com parâmetros fisiológicos (variabilidade da frequência cardíaca) e psicológicos (percepção de estresse e recuperação) mais bem estabelecidos na literatura, a fim de testar a hipótese de associação entre tais variáveis e carga de treinamento.

A modalidade futebol foi escolhida devido às grandes exigências físicas, motoras e cognitivas, assim como, por que, no Brasil, onde este esporte possui grande relevância social, as temporadas esportivas são permeadas por eventos competitivos extensos e importantes, o que limita a aplicação de atividades de recuperação e a adequada regeneração dos atletas.

Por fim, estudos que investiguem os efeitos das cargas de treinamento e os estados de estresse e recuperação em modalidades coletivas são escassos ${ }^{10}$, o que torna grande a relevância de pesquisas com esse tipo de modalidade.

\section{MATERIAL E MÉTODOS}

Participaram deste estudo 14 jogadores de futebol de campo do

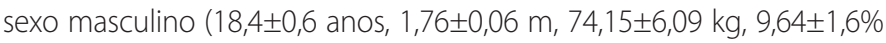
gordura, $49,4 \pm 2,8 \mathrm{ml} / \mathrm{kg} / \mathrm{min}$ de $\left.\mathrm{VO}_{2 \text { máx }}\right)$ pertencentes a um clube de primeira divisão do futebol brasileiro que competem em nível nacional e internacional na categoria júnior. Os atletas praticavam a modalidade há, em média, 10,7 1,5 anos. Compunham a amostra os 10 atletas titulares da equipe e quatro dos reservas, os quais participavam frequentemente dos jogos em situações de substituição.

Dos 14 voluntários, quatro foram excluídos da amostra devido a lesões ocorridas durante o período do estudo, que os impediram de permanecer treinando junto ao grupo. Para tal, foi considerado o afastamento superior a uma semana, pois, assim, eles não estariam mais submetidos às mesmas cargas de treinamento dos demais atletas.

O estudo respeitou todas as normas estabelecidas pela Comissão Nacional de Ética em Pesquisa (CONEP) por meio da Resolução 196/96 do Conselho Nacional de Saúde (CNS), para pesquisas que envolvem seres humanos e foi aprovado pelo Comitê de Ética em Pesquisa (COEP) da Universidade Federal de Minas Gerais, Brasil, obtendo o parecer no. ETIC 0579.0.203.000-09.

Realizou-se contato com a diretoria do clube e, após autorização, estabeleceu-se comunicação com a comissão técnica da equipe de juniores para elucidação dos métodos e procedimentos da pesquisa, e recrutamento dos atletas. Posteriormente, antes de se iniciarem as coletas, realizou-se uma reunião com os jogadores, na qual foram explicitados todos os procedimentos, possíveis riscos e benefícios do estudo. A seguir foi realizada a leitura do Termo de Consentimento Livre e Esclarecido - TCLE e após esse procedimento, abriu-se espaço 
para o esclarecimento de quaisquer dúvidas existentes. Os participantes então assinaram o TCLE declarando-se voluntários da pesquisa, e foram instruídos sobre a possibilidade de eles deixarem de participar do estudo, a qualquer momento, durante sua realização sem nenhuma perda ou constrangimento.

Foram realizadas coletas das variáveis estudadas em três momentos distintos, os quais se caracterizaram como:

A primeira coleta (Controle $=\mathrm{C}$ ) foi realizada após um período de treinamentos de três semanas considerado, pela comissão técnica, constituído por cargas de treinamento moderadas, que antecedia um novo momento, no qual haveria um aumento substancial dessas cargas.

A segunda coleta foi feita quatro semanas após a primeira, o que caracterizou o período considerado de maiores cargas de treinamento dentro do planejamento da comissão técnica (treinamento intenso =T1) . Nesse período houve aumento da duração e intensidade das sessões de treinamento e, este, foi composto por maior conteúdo físico e técnico.

A terceira coleta foi realizada quatro semanas após a segunda coleta. As cargas de treinamento foram reduzidas durante esse período (treinamento leve $=$ T2) no intuito de recuperar os atletas do período anterior. Além da redução na carga geral de treinamento, os atletas também foram dispensados por sete dias, nos quais puderam ir para casa e descansar junto a suas famílias. Esse período de treinamentos foi composto predominantemente por conteúdo tático.

Para registro e análise da carga de treinamento, foram captadas as frequências cardíacas de trabalho de todas as sessões de treinamento a partir da primeira coleta, que funcionou como linha de base. Esses

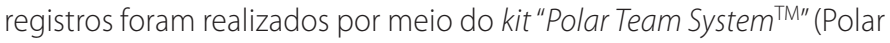
Electro Oy, Finlândia). Foram efetuados registros ao longo de todas as sessões de treinamento em oito atletas a cada sessão. Os oito voluntários, os quais teriam a frequência cardíaca de treino monitorada, foram revezados de forma aleatória a cada sessão de treinamento, o que garantiu que todos os indivíduos tivessem suas frequências cardíacas de treino registradas, durante diferentes sessões de treinamento, ao longo dos dois períodos de treinamento analisados, o que garante a representatividade dos registros para o monitoramento das cargas de treinamento.

A quantificação das cargas de treinamento foi feita através do método de impulsos de treinamento (TRIMP) proposto por Edwards 22 . Esse método baseia-se na multiplicação do número de minutos em que o indivíduo realiza exercício dentro de uma determinada faixa de intensidade de frequência cardíaca por valores de referência, que vão de um a cinco (50-60\% da $\mathrm{FC}_{\text {máx }}=1 ; 60-70 \%$ da $\mathrm{FC}_{\text {máx }}=2 ; 70-80 \%$ da $F C_{\text {máx }}=3 ; 80-90 \%$ da $F C_{\text {máx }}=4 ; 90-100 \%$ da $F C_{\text {máx }}=5$ ), de acordo com a zona de frequência cardíaca utilizada no exercício. Em seguida, somam-se os valores encontrados para cada zona e o resultado final é o número de impulsos de treinamento na sessão de treino.

Para encontrar a carga de treinamento individual dos atletas nos diferentes períodos de cargas de treinamento, calculou-se a média dos impulsos de treino das sessões em que o indivíduo teve sua frequência cardíaca de treino registrada e multiplicou-se esse valor pelo número de sessões de treinamento total do respectivo período de treinamento avaliado, como demonstrado na equação:

\section{CARGA = Média dos TRIMPs registrados $\times$ ST}

onde, ST corresponde ao número de sessões de treinamento realizadas ao longo do período de treinamentos avaliado.

Para verificar possíveis interferências de alterações climáticas sobre as respostas da frequência cardíaca utilizada para o cálculo dos TRIMPs nos dois períodos de treinamento analisados, mediu-se a temperatura ao longo dos treinos utilizando um termômetro de IBUTG (RS-214, WIBGET ${ }^{\circledR}$, EUA) em intervalos de 15 minutos ao longo de cada sessão de treino, de modo a se obter uma média da temperatura de cada sessão de treinamento e comparar as variações climáticas dos dois períodos de treinamento.

A versão em português do Brasil do Questionário de Estresse e Recuperação para Atletas (RESTQ-Sport) foi utilizada para medir a incidência de estresse e de atividades de recuperação. Este instrumento é composto por 77 itens, que são respondidos em uma escala tipo Likert de sete pontos a qual varia de $0=$ "nunca" a 6 = "sempre". Há um item introdutório e os demais 76 dividem-se em 19 escalas, das quais sete avaliam estresse geral, cinco recuperação geral, três estresse específico do esporte e quatro recuperação específica do esporte.

Essa versão do RESTQ-Sport possui validade e confiabilidade adequadas para a avaliação proposta ${ }^{18}$.

A frequência cardíaca de repouso foi registrada de maneira contínua por um intervalo de 10 minutos, caracterizando o registro de curta duração necessário para análise da VFC. Para tal, os atletas permaneciam deitados em decúbito dorsal, em local previamente preparado para esse procedimento. Eles eram orientados a permanecerem relaxados, de olhos fechados e acordados. Os voluntários também foram orientados para não fazer uso de bebidas alcoólicas ou qualquer produto que pudesse conter cafeína ou estimulantes nas 24 horas anteriores às coletas.

Ao longo das coletas dos registros de frequência cardíaca de repouso que foram usados para análise da VFC, também foi efetuado o registro das temperaturas nos três momentos $C$, T1 e T2. Foram realizadas medições de temperatura a cada cinco minutos, com o mesmo equipamento usado nas medidas durante os treinamentos, verificando-se, assim, possíveis diferenças nas temperaturas ambiente nos três momentos de coletas.

A frequência cardíaca foi registrada batimento-a-batimento (intervalos R-R) por meio de frequencímetro cardíaco - Polar Electro Oy / modelo VANTAGE NV (Polar ${ }^{\circledR}$, Finlândia) de maneira contínua e posteriormente transmitida para o computador por meio de interface modelo - Polar Advantage Interface System (Polar ${ }^{\circledR}$, Finlândia), utilizando o software "Polar HR Analysis" versão 5.04.03, para Windows (Polar Electro Oy, Kempele, Finlândia, 1995).

Os ciclos respiratórios efetuados pelos participantes da amostra durante os registros da FC de repouso para análise da VFC foram contados visualmente por meio das observações das incursões respiratórias. Para a marcação do tempo durante a contagem dos ciclos, foi utilizado um relógio digital simples. Em seguida foi calculado o número de ciclos respiratórios por minuto realizados ao longo dos registros.

Os arquivos das frequências cardíacas foram observados quanto à quantidade de erro presente em cada registro. Para essa verificação, foi usado o software "Polar Precision Performance SW", versão 4.03.040, para Windows (Polar Electro Oy, Kempele, Finlândia, 2005). Foram considerados apenas registros que obtiveram erro inferior a $2 \%$ no intervalo de tempo que seria analisado.

Para a VFC, analisaram-se intervalos de cinco minutos de menor variância entre os batimentos cardíacos consecutivos, utilizando o software "Kubios Heart Rate Variability" versão 2.0 para Windows (Biosignal Analysis and Medical Imaging Group, Departament of Physics, University of Kuopio, Kuopio, Finlândia). Inicialmente, os intervalos foram corrigidos utilizando um filtro moderado e interpolados a uma frequência de $4 \mathrm{~Hz}$, ou seja, até 4 pontos por minuto. Isso foi realizado para que a análise da VFC fosse feita considerando apenas iNNs (intervalos normal-normal) ${ }^{23}$, desconsiderando possíveis batimentos irregulares não provenientes da despolarização sinusal ou erro de medida. 
A VFC foi analisada no domínio da frequência: a potência das altas frequências ( $\mathrm{HF} ; 0,15-0,40 \mathrm{~Hz}$ ), a potência das baixas frequências ( $(\mathrm{F} ; 0,04-0,15 \mathrm{~Hz}$ ), a potência das muito baixas frequências ( $\mathrm{VLF} ; 0-0,04 \mathrm{~Hz}$ ), total de potencia (TP) e a relação LF/HF. E no domínio do tempo, utilizando-se os índices: Média FC (média da frequência cardíaca), Média R-R (média dos intervalos R-R), SDNN (desvio padrão dos INNs), RMSSD (raíz quadrada da média da diferença entre INNs consecutivos), NN50 (números de INN com diferenças sucessivas maiores que 50ms e pNN50 (porcentagem dos INNs nos quais as diferenças sucessivas entre eles são maiores do que $50 \mathrm{~ms})^{23}$.

Para a avaliação do tempo de reação simples (SRT), foi usado o equipamento para avaliação psicológica "Multipsy-821" (Bio-Data, Steinbach, Áustria).

O teste consiste de 21 estímulos luminosos idênticos emitidos em intervalos de tempo aleatórios os quais devem ser respondidos por meio de um botão, que deve ser pressionado no menor tempo possível sempre que um estímulo é fornecido. O indivíduo testado mantém-se ao longo de toda a execução do teste com o dedo indicador da mão preferencial posicionado sobre o botão de resposta, $\mathrm{e}$ a cada acendimento da luz (estímulo) ele aperta o botão no menor tempo possível. Para o cálculo da média do tempo de reação foram utilizadas respostas a estímulos concentradas entre $150 \mathrm{~ms}$ e 1,5 s de tempo de reação, considerando respostas inadequadas as quais se encontravam fora desse intervalo. $\mathrm{O}$ teste possui um coeficiente de fidedignidade adequado para medir o tempo de reação de acordo com o fabricante.

O teste de tempo de reação era realizado em uma sala com o mínimo de ruídos, na qual permaneciam apenas o atleta testado e o pesquisador responsável pela aplicação do teste. $\mathrm{O}$ aplicador dos testes realizava um procedimento-padrão com todos os voluntários, determinando a posição em que os mesmos deveriam permanecer sentados e explicando a realização do teste por meio de instrução padronizada e esclarecendo possíveis duvidas que ocorressem.

As coletas foram realizadas sempre no horário da manhã entre as oito e 11 horas, com o intuito de se prevenirem interferências do ciclo circadiano sobre as variáveis. A coleta se iniciava aproximadamente uma hora após o despertar dos atletas, momento gasto para troca de roupas e desjejum.

Todas as coletas foram realizadas no próprio clube, em um auditório e em salas do departamento médico. Utilizaram-se esses ambientes para que se conseguisse o máximo de tranquilidade e privacidade, necessárias para os voluntários. Permaneceram nos locais de coletas durante os procedimentos apenas os atletas participantes e os pesquisadores envolvidos.

\section{Análise estatística}

Inicialmente, realizou-se a análise descritiva dos dados, composta de medianas, médias \pm desvios-padrão. Em seguida, realizou-se o teste de Shapiro-Wilk para testar a hipótese de distribuição normal das variáveis.

Para as variáveis que apresentaram distribuição normal, adotou-se estatística paramétrica. Para verificar diferenças entre C, T1 e T2, foi feita análise de variância (ANOVA two-way). Além disso, foi realizado o teste de Levene para verificar a homogeneidade das variâncias de amostras independentes.

Quando as distribuições não foram normais, adotou-se estatística não paramétrica, utilizando teste de Friedman como análise de variância para verificar diferenças entre os períodos $C, T 1$ e T2, seguido do teste de Wilcoxon para identificar onde se encontravam as diferenças. Porém houve situações em que variáveis investigadas não apresentaram distribuição normal em apenas um dos momentos de coleta, o que indicou a utilização do teste de Friedman como análise de variância. Mas, para análises múltiplas, foi realizado o teste de Wilcoxon para as comparações que envolviam a variável não normal e teste " $t$ " pareado para comparar as variáveis de distribuição normal.

\section{RESULTADOS}

Inicialmente destaca-se que não houve diferença significativa $(p=0,452)$ nas médias de temperaturas dos períodos $\mathrm{T} 1\left(27,4 \pm 3^{\circ} \mathrm{C}\right)$ e T2 $\left(26,8 \pm 2,5^{\circ} \mathrm{C}\right)$, entre as temperaturas das sessões de treinamento do primeiro para o segundo período. Assim acredita-se que possíveis modificações nos impulsos de treinamento, resultantes das medidas de FC durante os treinamentos, derivam de reais modificações nas cargas de treinamento e nas atividades desenvolvidas ao longo dos treinos, uma vez que a possibilidade de os atletas terem vivenciado períodos de treinamentos com temperaturas diferentes é descartada quando comparadas as médias de temperaturas de T1 e T2.

Identificou-se diferença significativa $(p=0,014)$ entre os impulsos de treinamento nos dois períodos de coletas, sendo T1 $(2504,7 \pm 572,6$ TRIMPs) > T2 (2177,2 \pm 558,6 TRIMPs).

A figura 1 mostra as médias e os desvios-padrão dos escores obtidos pelo grupo de atletas ao longo da temporada (C, T1 e T2), o que representa os estados de estresse e recuperação apresentados pelos

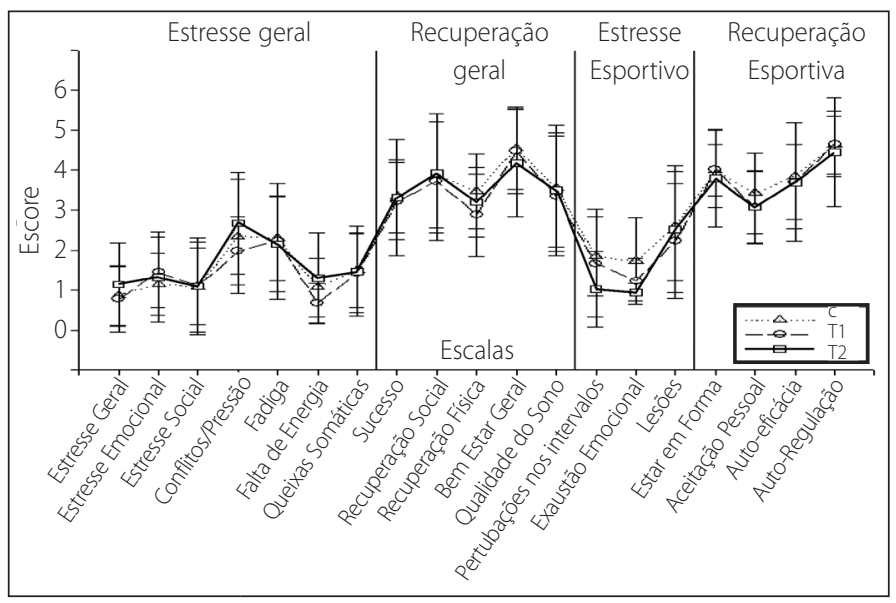

Figura 1. Percepção de estresse e recuperação em C, T1 e T2 em 19 escalas, segundo o REST-Q Sport $(n=10)$.

atletas. Não foi encontrada diferença significativa nos três momentos de coleta de acordo com a análise de variância $(p>0,05)$.

As temperaturas durante as coletas tiveram médias e desvios-padrão de 22,9 $\pm 0,5^{\circ} \mathrm{C}$ (Controle), $22,9 \pm 0,3^{\circ} \mathrm{C}$ (Treinamento intenso) e 23,9 $\pm 0,2^{\circ} \mathrm{C}$ (Treinamento leve), oscilando entre mínima de $22,5^{\circ} \mathrm{C}$ em C e máxima de $24,3^{\circ} \mathrm{C}$ em T2. As médias e desvios-padrão dos ciclos respiratórios por minuto realizados ao longo das coletas da FCrep para análise da VFC foram de 18,1 $\pm 2,7$ em C, 17,6 $\pm 2,9$ em T1 e 17,7 $\pm 2,6$ em T2. Não se encontrou diferença entre o número de ciclos respiratórios por minuto nas três coletas realizadas $(p=0,93)$.

Os valores de medianas, médias e desvios-padrão dos índices de VFC encontrados no estudo são apresentados na tabela 1. Os valores de VFC não se alteraram significativamente $(p>0,05)$ ao longo das coletas.

\section{Tempo de reação}

A análise de variância mostrou que houve diferença significativa entre as coletas $(p=0,02)$. Em seguida, as comparações múltiplas mostraram que a diferença existente localiza-se entre C e T1 $(p=0,047)$, sendo $\mathrm{T} 1(230,4 \pm 48,9 \mathrm{~ms})>C(212,4 \pm 25,5 \mathrm{~ms})$, como apresentado na figura 2. 
Tabela 1. Variabilidade da frequência cardíaca em C, T1 e T2 ( $=10)$.

\begin{tabular}{c|c|c|c|c|c|c|c|c|c}
\hline \multirow{2}{*}{ Índices de VFC } & \multicolumn{3}{|c|}{ C } & \multicolumn{3}{c|}{ T1 } & \multicolumn{2}{c}{ T2 } \\
\cline { 2 - 10 } & Mediana & Média & DP & Mediana & Média & DP & Mediana & Média & DP \\
\hline VLF (0-0.04 Hz)ms2 & 2306 & 2875,5 & 2348,5 & 1018 & 2379,2 & 2893,7 & 1419,5 & 5136,3 & 8673,8 \\
\hline LF (0.04-0.15 Hz)ms2 & 1566,5 & 2348,2 & 2040,6 & 1476,5 & 2873,3 & 2914,3 & 1042 & 2679,6 & 3908,9 \\
\hline HF (0.15-0.4 Hz)ms2 & 1629,5 & 1859,5 & 1471,9 & 3003,5 & 3000,9 & 1916,7 & 2268,5 & 3089,7 & 2209,9 \\
\hline TP (ms2) & 5944 & 7083,3 & 5053,5 & 5935,5 & 8253,5 & 6856,8 & 5979 & 10905,5 & 11944,2 \\
\hline LF (N.U.) & 48,5 & 51,2 & 15,5 & 39,9 & 42,8 & 18,1 & 33,9 & 38 & 17,8 \\
\hline HF (N.U.) & 51,5 & 48,8 & 15,5 & 60,1 & 57,2 & 18,1 & 66,2 & 62 & 17,8 \\
\hline LF/HF (ms2) & 1 & 1,6 & 2,1 & 0,7 & 0,9 & 0,7 & 0,5 & 0,7 & 0,6 \\
\hline Média FC & 56,3 & 56,08 & 6,8 & 50,7 & 49,7 & 6,3 & 50,0 & 49,9 & 7,1 \\
\hline Média R-R & 1076,6 & 1092,2 & 135,5 & 1190,6 & 1233,4 & 160,7 & 1212,1 & 1235,1 & 188,7 \\
\hline SDNN & 78 & 84,7 & 30,0 & 77,3 & 87 & 32,6 & 81,2 & 95,4 & 48,3 \\
\hline RMSSD & 68,1 & 78,9 & 1,7 & 85,8 & 100,1 & 37,1 & 88,2 & 103,3 & 38,7 \\
\hline NN50 & 137 & 125,3 & 53,6 & 146 & 147,1 & 41,4 & 139,5 & 149,2 & 46,9 \\
\hline pNN50 & 46,9 & 45,9 & 21,7 & 60,9 & 59,9 & 16,9 & 60,4 & 60,3 & 16,8 \\
\hline
\end{tabular}

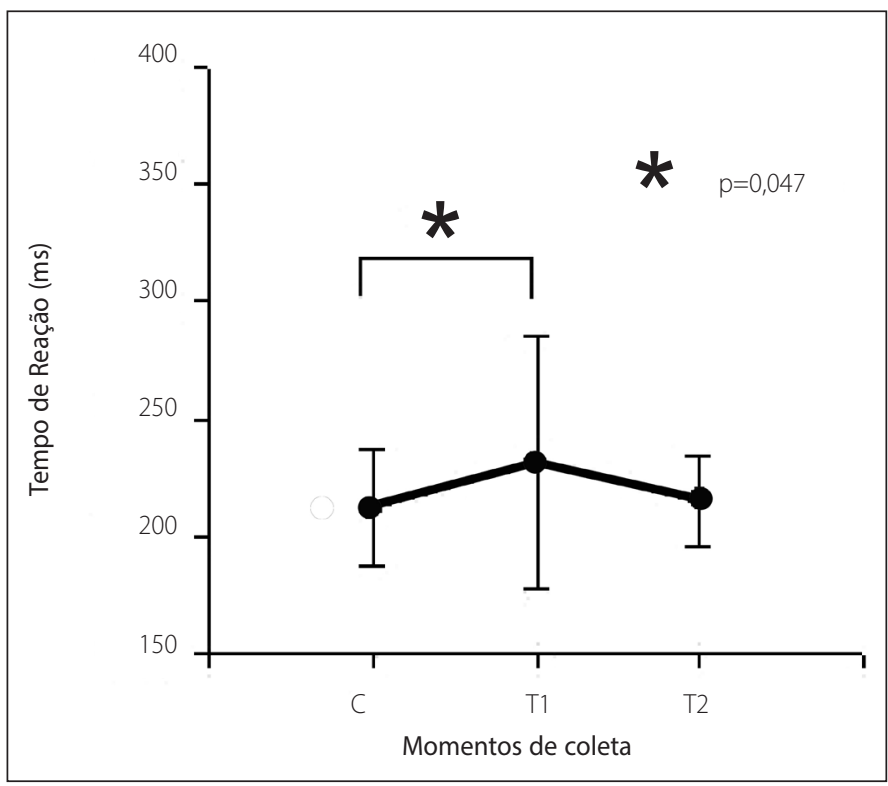

Figura 2. Médias dos tempos de reação em C, T1 e T2 (ms) ( $n=10)$.

\section{DISCUSSÃO}

Durante as três coletas de dados, não houve alteração na percepção de estresse e recuperação (RESTQ-Sport) e na VFC, sendo que os escores encontrados pelo questionário indicam baixos níveis de estresse e bons níveis de recuperação. Isso pode ter ocorrido porque o aumento das cargas de treinamento pode não ter tido magnitude tal que houvesse modificação na percepção de estresse e recuperação e na VFC dos indivíduos após T1 (treinamento intenso), permanecendo os atletas com níveis adequados de estresse e recuperação nesse período e, consequentemente, em T2, no qual os treinos foram mais leves.

A maioria dos estudos que utilizaram o RESTQ-Sport para medir os níveis de estresse e recuperação ao longo de temporadas esportivas e de períodos específicos do treinamento encontra alterações significativas nos estados de estresse e recuperação em diferentes períodos de treinamento 5,7,8,11,12,20,21. Essas alterações, por sua vez, normalmente, assumem uma característica de dose-resposta em relação às cargas de treinamento. Isto é, elevações nas cargas de treinamento geram elevações nos níveis de estresse e redução nos níveis de recuperação.

No entanto, há estudos em que o referido instrumento não foi capaz de detectar as mudanças de cargas de treinamento. Purge et al. ${ }^{24}$ não encontraram alterações significativas nos níveis de estresse e recuperação de 11 remadores ao longo de vinte e quatro semanas de treinamento. Em seu estudo, os autores justificaram o observado como sendo devido aos volumes de treinamento terem variado pouco, o que não causou mudanças na percepção dos atletas. Já González-Boto et al. ${ }^{20}$ também não encontraram diferenças significativas ao aumentar as intensidades de treinamento de nove nadadores de elite. Nesse caso, os pesquisadores fundamentaram este resultado com o fato de que, ao se elevarem as intensidades dos treinamentos, houve uma redução substancial dos volumes nadados durante o período.

Em ambos os estudos foram utilizados, como forma de controle das cargas de treinamento, medidas de distância e velocidade, o que não necessariamente representaria mudanças nas cargas totais a que eram submetidos os atletas, uma vez que, no planejamento do treinamento, considerar-se uma tendência de relação inversa entre volume e intensidade pode ser considerada uma estratégia para controlar o excesso de treinamento ${ }^{25}$. Assim, no presente estudo, ao se utilizar como medida das cargas de treinamento os TRIMPs, cria-se um escore representante de ambos os componentes da carga de treinamento e considera-se que as cargas foram significativamente diferentes T1 > T2. Entretanto, os dados de carga de treinamento medidos através dos TRIMPs no presente estudo, não permitem inferências acerca da magnitude do aumento das cargas de treinamento de $C$ para T1, uma vez que não houve registros de frequência cardíaca para a quantificação dos TRIMPs nas semanas que antecederam C. Tal observação está em consonância com a ausência de alterações na percepção de estresse e recuperação, avaliada pelo REST-Q Sport.

Quanto à hipótese da VFC não ter se alterado devido à pequena magnitude no aumento das cargas de treinamento em T1, em estudos nos quais houve aumentos consideráveis das cargas de treinamento, da ordem de até $100 \%$ do total treinado ${ }^{13,14}$, foram observadas mudanças significativas na VFC. Contudo, estudos recentes em que as alterações das cargas de treinamento não foram tão grandes, não encontraram alterações na VFC após períodos com cargas de treinamento diferentes, moderadas e altas ao longo da temporada esportiva ${ }^{16,17}$.

Sendo assim, mudanças na modulação autonômica cardíaca aparentemente são percebidas apenas em situações em que atletas são submetidos a cargas de treinamento extremamente elevadas, de forma a levar os voluntários à exaustão9,15, o que nem sempre ocorre numa situação real de treinamento com finalidade competitiva, como nesta investigação, na qual o programa de treinamento foi concebido visando a participação em competição de nível nacional, e, não, no intuito de levar os voluntários a situações extremas de fadiga crônica. Outros estudos mostram, ainda, que ocorrem modificações na atuação simpática apenas em períodos curtos de tempo como adaptação ao treinamento, imediatamente seguintes às alterações na carga de exercícios ${ }^{13,14,17}$. No presente estudo as coletas foram realizadas em intervalos de quatro 
semanas, o que pode ter permitido aos atletas uma adaptação às novas cargas de treinamento ao longo deste período.

De um modo geral os valores da VFC foram considerados bons já que houve uma predominância da atividade vagal, a qual é considerada positiva para a saúde cardiovascular tanto de atletas quanto de jovens, adultos e idosos ${ }^{14,26,27}$, indicando que as cargas de treinamento estavam adequadas e que os indivíduos eram bem condicionados aerobicamente.

Embora os atletas aparentemente estivessem bem treinados, devido aos bons estados de estresse e recuperação (RESTQ-Sport) e aos bons valores de VFC, o tempo de reação foi significativamente maior após T1, mostrando que essa variável é sensível às alterações nas cargas de treinamento, o que indica ser esta variável um marcador relevante para o monitoramento das cargas de treinamento esportivo, principalmente em períodos de treinamentos extenuantes, como sugerido em outros estudos 1,4,19,28.

Contudo, não houve redução dos tempos de reação após T2, o que demonstra que a variável não foi sensível à redução de cargas de treinamento. Entretanto, a recuperação não depende apenas da redução das cargas de treinamento, mas também de outros fatores, tais como atividades regenerativas, alimentação adequada, técnicas de redução de estresse, biofeedback e estratégias de coping ${ }^{29}$, as quais podem ter sido insuficientes ou inadequadas para a recuperação dos atletas e que não foram controladas no presente trabalho.

Diversos estudos têm encontrado déficits em funções executivas em resposta a treinamento extenuante 1,4,6,719,28, as quais, por sua vez, têm sido associadas à VFC, sendo uma maior variabilidade relacionada à melhor condição cognitiva, maior capacidade adaptativa e melhor desempenho em tarefas executivas ${ }^{29}$. Entretanto, tarefas não-executivas, como o tempo de reação simples, não apresentam a mesma associação com a VFC, pois uma reduzida VFC pode estar associada a estados de alerta, alta excitabilidade e velocidade de resposta em tarefas que não exigem tomadas de decisão ${ }^{29}$.

No presente estudo não foram observadas alterações significativas na VFC, mas ocorreu um aumento do tempo de resposta, o que pode indicar que as funções não-executivas são mais diretamente influenciadas por mecanismos de fadiga central, neurotransmissão inadequada ${ }^{28,30}$ do que pelo controle inadequado do fluxo sanguíneo no cérebro pelo sistema nervoso autônomo ${ }^{29}$. O déficit no tempo de reação encontrado neste estudo possibilita inferir que esta seja uma função cognitiva, a qual sofre danos consideráveis em períodos de treinamento físicos extenuantes, sendo que estas alterações ocorrem anteriormente às modificações nas funções executivas, fisiológicas, à percepção subjetiva de determinados estados psicológicos, a aspectos comportamentais e afetivos, sugerido ser essa variável seja uma ferramenta importante para detectar estados de overreaching e overtraining ainda em estágios iniciais.

\section{CONCLUSÃO}

Conclui-se que altas cargas de treinamento provocaram lentidão psicomotora. E que, a VFC e a percepção de estresse e recuperação, não foram sensíveis às alterações das cargas de treinamento encontradas neste estudo, em situação real de treinamento durante uma temporada competitiva.

Possivelmente o tempo de reação possa ser, dentre os parâmetros utilizados, o mais sensível para a identificação de possíveis prejuízos no desempenho esportivo, oriundos de excessivas exigências de treinos e competições durante períodos competitivos, nas quais nem sempre há uma variação muito acentuada nas cargas de treinamentos.

Todos os autores declararam não haver qualquer potencial conflito de interesses referente a este artigo.

\section{REFERÊNCIAS}

1. Nederhof E, Lemmink K, Zwerver J, Mulder T. The effect of high load training on psychomotor speed. Int J Sports Med. 2007;28(7):595-601.

2. Hynynen E, Uusitalo A, Konttinen N, Rusko H. Heart rate variability during night sleep and after awakening in overtrained athletes. Med Sci Sports Exerc. 2006;38(2):313-7.

3. Smith DJ. A framework for understanding the training process leading to elite performance. Sports Med. 2003;33(15):1103-26.

4. Hynynen E, Uusitalo A, Konttinen N, Rusko H. Cardiac autonomic responses to standing up and cognitive task in overtrained athletes. Int J Sports Med. 2008;29(7):552-8.

5. Steinacker JM, Lormes W, Kellmann M, Liu Y, Reissnecker S, Opitz-Gress A, et al. Training of junior rowers before world championships. Effects on performance, mood state and selected hormonal and metabolic responses. J Sports Med Phys Fitness. 2000;40(4):327-35.

6. Nederhof E, Lemmink KA, Visscher C, Meeusen R, Mulder T. Psychomotor speed: possibly a new marker for overtraining syndrome. Sports Med. 2006;36(10):817-28.

7. Nederhof E, Zwerver J, Brink M, Meeusen R, Lemmink K. Different diagnostic tools in nonfunctional overreaching. Int J Sports Med. 2008;29(7):590-7.

8. Kellmann M, Altenbur D, Steinacker Jm, Lornes W. Assessing stress and recovery during preparation for the world championships in rowing. Sport Psychol. 2001;15:151-67.

9. Baumert M, Brechtel L, Lock J, Hermsdorf M, Wolff R, Baier V, et al. Heart rate variability, blood pressure variability, and baroreflex sensitivity in overtrained athletes. Clin J Sport Med. 2006;16(5):412-7

10. Maso F, Lac G, Filaire E, Michaux O, Robert A. Salivary testosterone and cortisol in rugby players: correlation with psychological overtraining items. Br J Sports Med. 2004;38(3):260-3

11. Coutts AJ, Wallace LK, Slattery KM. Monitoring changes in performance, physiology, biochemistry, and psychology during overreaching and recovery in triathletes. Int J Sports Med. 2007;28(2):125-34.

12. Filaire E, Rouveix M, Duclos M. Training and 24-hr urinary catecholamine excretion. Int J Sports Med. 2009;30(1):33-9

13. Uusitalo AL, Uusitalo AJ, Rusko HK. Endurance training, overtraining and baroreflex sensitivity in female athletes. Clin Physiol. 1998;18(6):510-20.

14. lellamo F, Legramante JM, Pigozzi F, Spataro A, Norbiato G, Lucini D, et al. Conversion from vagal to sympathetic predominance with strenuous training in high-performance world class athletes. Circulation. 2002;105(23):2719-24.

15. Hansen AL, Johnsen BH, Sollers JJ 3rd, Stenvik K, Thayer JF. Heart rate variability and its relation to prefrontal cognitive function: the effects of training and detraining. Eur J Appl Physiol. 2004;93(3):263-72.
16. Vinet A, Beck L, Nottin S, Obert P. Effect of intensive training on heart rate variability in prepubertal swimmers. Eur J Clin Invest. 2005;35(10):610-4.

17. Perini R, Tironi A, Cautero M, Di Nino A, Tam E, Capelli C. Seasonal training and heart rate and blood pressure variabilities in young swimmers. Eur J Appl Physiol. 2006;97(4):395-403.

18. Costa LOP, Samulski DM. Processo de validação do Questionário de Estresse e Recuperação para Atletas (RESTQ-Sport) na Língua Portuguesa. R Bras Ci Mov. 2005;13(1):79-86.

19. Rietjens GJ, Kuipers H, Adam JJ, Saris WH, van Breda E, van Hamont D, et al. Physiological, biochemical and psychological markers of strenuous training-induced fatigue. Int J Sports Med. 2005;26(1):16-26.

20. González-Boto R, Salguero A, Tuero C, González-Gallego J, Márquez S. Monitoring the effects of training load changes on stress and recovery in swimmers. J Physiol Biochem. 2008;64(1):19-26.

21. Jürimäe J, Mäestu J, Purge $P$, Jürimäe T. Changes in stress and recovery after heavy training in rowers J Sci Med Sport. 2004;7(3):335-9.

22. Edwards S. High performance training and racing. In: Edwards S, editor. Sacramento, CA: Feet Fleet Press; 1993. p. 113-123.

23. Heart rate variability: standards of measurement, physiological interpretation and clinical use. Task Force of the European Society of Cardiology and the North American Society of Pacing and Electrophysiology. Circulation. 1996;93(5):1043-65.

24. Purge $P$, Jürimäe J, Jürimäe T. Hormonal and psychological adaptation in elite male rowers during prolonged training. J Sports Sci. 2006;24(10):1075-82

25. Uusitalo AL. Overtraining: making a difficult diagnosis and implementing targeted treatment. Phys Sportsmed. 2001;29(5):35-50.

26. Gamelin FX, Berthoin S, Sayah H, Libersa C, Bosquet L. Effect of training and detraining on heart rate variability in healthy young men. Int J Sports Med. 2007;28(7):564-70.

27. Pichot V, Roche F, Denis C, Garet M, Duverney D, Costes F, et al. Interval training in elderly men increases both heart rate variability and baroreflex activity. Clin Auton Res. 2005;15(2):107-15.

28. Dupuy $\mathrm{O}$, Renaud $M$, Bherer $L$, Bosquet $L$. Effect of functional overreaching on executive functions. Int J Sports Med. 2010;31(9):617-23..

29. Thayer JF, Hansen AL, Saus-Rose E, Johnsen BH. Heart rate variability, prefrontal neural function, and cognitive performance: the neurovisceral integration perspective on self-regulation, adaptation, and health. Ann Behav Med. 2009;37(2):141-53.

30. Meeusen R, Watson $P$, Hasegawa $H$, Roelands B, Piacentini MF. Brain neurotransmitters in fatigue and overtraining. Appl Physiol Nutr Metab. 2007;32(5):857-64. 